predominantly due to the fact that the NHMRC standards are based on physically published guidelines.

Discussion A model of rapid guideline updates utilising a wiki platform is able to accommodate robust methodology and meets most of the current Australian standards.

\section{WHAT DO GUIDELINE APPRAISAL TOOLS ASSESS? A SYSTEMATIC REVIEW}

${ }^{1} U$ Siering, ${ }^{2} M$ Eikermann, ${ }^{1} E$ Hausner, ${ }^{1} W$ Hoffmann-Eber, ${ }^{2} E$ Neugebauer. ${ }^{1} I n s t i t u t e$ for Quality and Efficiency in Health Care (IQWiG), Cologne, Germany; ${ }^{2}$ Institute for Research in Operative Medicine (IFOM), Witten-Herdecke University, Cologne, Germany

\section{0:1136/bmjgs-2013-002293.66}

Background Clinical practice guidelines should provide a rational basis for healthcare decisions; however, their quality is often poor.

Objectives To systematically identify and describe guideline appraisal tools and draw conclusions for guideline appraisal.

Methods We conducted a systematic search in MEDLINE, EMBASE and the Cochrane Library for English and German-language guideline appraisal tools published after 1995. Reference lists of included publications were also screened. Dimensions of guideline quality were then generated from these publications and from articles by Cluzeau 1999, Graham 2000 and Vlayen 2005. Finally, the questions contained in the appraisal tools were allocated to the quality dimensions and summarised.

Results Overall, 40 appraisal tools were included and 13 quality dimensions identified. The main focus was the identification, assessment and presentation of evidence in guidelines. Questions on dealing with norms and values in guideline development, patient involvement, conflicts of interest, or implementation of guidelines into clinical practice were rare. The tools often assessed the appropriate documentation of the guideline development process (e.g. reporting of the search strategy), without addressing the appraisal of content of the development process (e.g. appropriateness of the search strategy) and the appraisal of clinical content.

Discussion Because many appraisal tools do not contain questions on norms and values or on potential conflicts of interest of guideline authors, important aspects potentially influencing the reliability of guidelines are not covered. In addition, an appraisal of content of the guideline development process and an appraisal of clinical content are often lacking.

\section{DEVELOPMENT OF A STATEWIDE GUIDELINES PROGRAM USING THE ADAPTE FRAMEWORK}

${ }^{1,3} \mathrm{~V}$ King, ${ }^{1} \mathrm{~S}$ Vandegriff, ${ }^{1,3} \mathrm{~A}$ Little, ${ }^{2} \mathrm{D}$ Coffman, ${ }^{2,3} \mathrm{C}$ Livingston, ${ }^{2} \mathrm{~W}$ Shaffer, ${ }^{2} \mathrm{~J}$ Gingerich. ${ }^{1}$ Center for Evidence-based Policy, Oregon Health \& Science University, Portland, USA; ${ }^{2}$ Office of Oregon Health Policy and Research, Oregon Health Authority, Salem, USA; ${ }^{3}$ Department of Family Medicine, Oregon Health \& Science University, Portland, USA

\section{0:1136/bmjgs-2013-002293.67}

Background The legislature passed comprehensive health reform legislation in 2009 directing the state to "set standards for safe and effective care", including development of "best practice guidelines and standards that can be uniformly applied across public and private health care".

Objectives Develop guideline methodology and guidelines for statewide clinicians and payers.
Methods Employed ADAPTE framework for guideline development. Initial guidelines selected for development included three low back pain (LBP) topics: general evaluation and management of LBP (results described below), advanced imaging for LBP, and percutaneous interventions for LBP. Existing (seed) guidelines identified by searching 17 databases. Quality evaluated using modified AGREE II instrument. Multidisciplinary guideline development group selected and adapted seed guidelines. Stakeholder, peer review and public comments were solicited.

Results Thirteen seed guidelines were identified and 10 met minimal inclusion criteria for LBP evaluation and management topic. Dual quality rating found five of good or fair quality. Final seed guideline selected based on quality and scope. Key recommendations were adopted for state Medicaid programme, including conservative and chiropractic care only in first month and no advanced imaging without clinical "red flags". A consumer booklet was developed and distributed to consumer, provider, and payer groups. Over 2500 booklets were distributed, with over 11,000 page views on the website. The initial guideline process took over one year to complete.

Discussion Starting a new multi-stakeholder guideline development programme requires substantial investments of methodological expertise, staff time, funds and political capital, but can substantially impact state health policy decisions.

\section{UPDATING AN ADAPTED CPG: WHEN IS ENOUGH ENOUGH?}

${ }^{1} \mathrm{C}$ Harstall, ${ }^{1} \mathrm{C}$ Moga, ${ }^{1} \mathrm{~A}$ Scott, ${ }^{2} \mathrm{P}$ Taenzer, ${ }^{3} \mathrm{~T}$ Findlay. ${ }^{1}$ Institute of Health Economics, Edmonton, Canada; ${ }^{2}$ University of Calgary, Calgary, Canada; ${ }^{3}$ Alberta Health Services, Calgary, Canada

\section{0:1136/bmjqs-2013-002293.68}

Background Within 2 years of releasing a low back pain clinical practice guideline (CPG), the Alberta Ambassador Guideline Adaptation Programme was required to update its adapted guideline. No guidance or 'how to' manuals were located.

Objectives To develop a process for updating an adapted guideline. To expedite the process by determining which components can be removed without compromising rigour.

Methods CPGs and systematic reviews published since the release of the CPG were identified and appraised, and discordant and new recommendations were tabulated. The Guideline Development Group (GDG) was surveyed to identify new interventions of interest. Evidence from systematic reviews was included for 'do not know' recommendations and new interventions.

Results The original guideline had 50 recommendations, eight of which were in the 'do not know' category. This expanded to 85 recommendations in the update: 43 unchanged, 32 on GDGnominated new interventions, and 10 revised. The updated CPG has 33 'do not know' recommendations. One of the original eight 'do not know' recommendations was changed based on new evidence.

Discussion The challenge of maintaining the integrity and high standards of the original guideline meant that the update consumed more time and resources than planned. Clearly, some components of the process can be jettisoned without jeopardising the methodological rigour and comprehensiveness of the final product.

Implications The next update will be streamlined, including only new seed guidelines that meet the quality criteria of the 